\title{
Neutron Slowing Down with Inelastic Scattering
}

\author{
S. A. El Wakil, H. M. Machali \\ Physics Department, Faculty of Science, Mansoura University, Mansoura, Egypt, \\ and
}

E. A. Saad

Atomic Energy Establishment, Cairo, Egypt

Z. Naturforsch. 33a, 1452-1454 (1978); received September 16, 1978

\begin{abstract}
The neutron slowing-down equation in an infinite homogeneous medium with isotropic scattering is solved. The slowing-down kernel is separated into an elastic and an inelastic part. The collision density is expressed in terms of Green's function of the elastic scattering only. The GreulingGoertzel (G-G) approximation is used for the elastic scattering kernel, while Volkin's model is used for the inelastic one. A differential-difference equation for a one-level excited state is solved by Laplace transform. Discussion of the poles obtained in the Laplace inverse shows that there are forbidden zones in which there is no solution. Numerical calculations of the collision density in $\mathrm{Fes}^{6}$ at 922 and $865 \mathrm{kev}$ levels are performed, which give the same behaviour as obtained by Corngold. The average slowing-down time calculated with our approach agrees with Williams's result in the asymptotic solution.
\end{abstract}

The inclusion of inelastic scattering in neutron scattering has been performed by scattering kernel approximation based on continuous slowing down theory [1], evaporation model [2], and synthesis technique [3]. Other techniques using a more accurate treatment of inelastic scattering were developed by Syros [4], Premuda [5], Segev [6], Beynon [7], and El-Wakil [8].

In this work the scattering kernel in the homogeneous medium is separated into elastic and inelastic parts. Volkin's model is used for inelastic scattering while the Greuling Goertzel approximation is used for the elastic scattering. The collision density of the system is expressed in terms of the Green's function of elastic scattering only. A differential-difference equation for one level excited state is solved by Laplace transform. Numerical calculations of the collision density in $\mathrm{Fe}^{56}$ at 922 and $865 \mathrm{keV}$ levels are performed. The average slow ng-down time is also calculated, which coinicides with that of Williams [11] in the asymptotic approximation.

Let us now consider the integral equation which describes the neutron slowing down in an infinite homogeneous medium as

$$
(I-A) \psi(E)=S(E)
$$

where $I$ is the identity operator and $A$ is the slowing down integral operator:

$$
A \psi=\int \mathrm{d} E^{\prime} h_{\mathrm{s}}\left(E^{\prime}\right) f\left(E^{\prime} \rightarrow E\right) \psi\left(E^{\prime}\right)
$$

where

$$
h_{\mathrm{s}}\left(E^{\prime}\right)=\Sigma_{\mathrm{s}}(E) / \Sigma_{\mathrm{t}}(E)
$$

and $\psi$ is the collision density.

If the scattering kernel $f\left(E^{\prime} \rightarrow E\right)$ consists of an elastic and an inelastic part i.e.

$$
f\left(E^{\prime} \rightarrow E\right)=f_{\mathrm{e}}\left(E^{\prime} \rightarrow E\right)+f_{\text {in }}\left(E^{\prime} \rightarrow E\right),
$$

then Eq. (1) becomes

$$
\left(I-A_{\mathrm{e}}\right) \psi(E)-A_{\text {in }} \psi(E)=S(E)
$$

or

$\psi(E)-\left(I-A_{\mathrm{e}}\right)^{-1} A_{\text {in }} \psi(E)=\left(I-A_{\mathrm{e}}\right)^{-1} S(E) .(5$

The inversion $\left(I-A_{\mathrm{e}}\right)^{-1}$ is possible in terms of Green's function $G\left(E, E_{0}\right)$, i.e.

$$
\left(I-A_{\mathrm{e}}\right) G\left(E, E_{0}\right)=\delta\left(E-E_{0}\right),
$$

and thus Eq. (5) can be written in terms of Green's function of elastic scattering $G\left(E, E_{0}\right)$ as:

$$
\begin{aligned}
\psi(E)= & \int_{E}^{E_{0}} G\left(E^{\prime}, E\right) A_{\text {in }} \psi\left(E^{\prime}\right) \\
& +\int_{E}^{E_{0}} G\left(E^{\prime}, E\right) S\left(E^{\prime}\right) \mathrm{d} E^{\prime} .
\end{aligned}
$$

The Green's function for elastic scattering can be calculated for the $G-G$ approximation by a method adopted from Ref. [11] to give, upon neglecting the contribution of the non-collided term,

$$
G\left(E, E_{0}\right)=\frac{\xi h_{\mathrm{e}}}{\gamma^{2} E} \frac{I}{\left[I-\left(I-\frac{\xi}{\gamma}\right) h_{\mathrm{e}}\right]^{2}}\left(\frac{E_{0}}{E}\right)^{\beta}
$$


with

$$
\beta=\left(\frac{1}{\gamma}-I\right)-\frac{\xi h_{\mathrm{e}}}{\gamma^{2}\left[I-\left(I-\frac{\xi}{\gamma}\right) h_{\mathrm{e}}\right]} .
$$

Upon using Volkin's model

$$
f_{\text {in }}\left(E^{\prime} \rightarrow E\right)=\sum_{k=1}^{N} \delta\left(E^{\prime} \rightarrow E-E_{k}\right)
$$

for inelastic scattering in Eq. (7), it can be transformed by differentiating both sides with respect to $E$ to the differential-difference equation

$$
\begin{aligned}
\frac{\mathrm{d}}{\mathrm{d} E} \psi(E)+ & G\left(E, E_{0}\right) h_{\mathrm{in}}\left(E, E_{k}\right) \psi\left(E+E_{k}\right) \\
= & -G\left(E, E_{0}\right) S(E) .
\end{aligned}
$$

This equation is solved by using Laplace transform to give

$$
\psi(\varepsilon)=\frac{I}{2 \pi i} G\left(\varepsilon_{k}, \varepsilon_{0}\right) \int \frac{\mathrm{d} \lambda S(\lambda) e^{-\lambda \varepsilon}}{\lambda-h_{\mathrm{in}} G\left(\varepsilon_{k}, \varepsilon_{0}\right) e^{-\lambda \varepsilon_{k}}} .
$$

where $\varepsilon=E / E_{1}$.

The poles of this integral occur at

$$
\lambda-G\left(\varepsilon_{k}, \varepsilon_{0}\right) h_{\mathrm{in}} e^{-\lambda \varepsilon_{k}}=0 .
$$

Graphical solution of this equation for $\lambda=\sigma+i \tau$ shows that roots can only occur in the following band of positive $\tau$ :

$$
\frac{(2 n+1) \pi}{\varepsilon_{k}}<\tau<\frac{(2 n+2) \pi}{\varepsilon_{k}} .
$$

An analogous expression holds for negative values of $\tau$.

One can also show that at least one and at most three roots occur in each allowed band. It is easily shown [12] that the allowed band roots must have

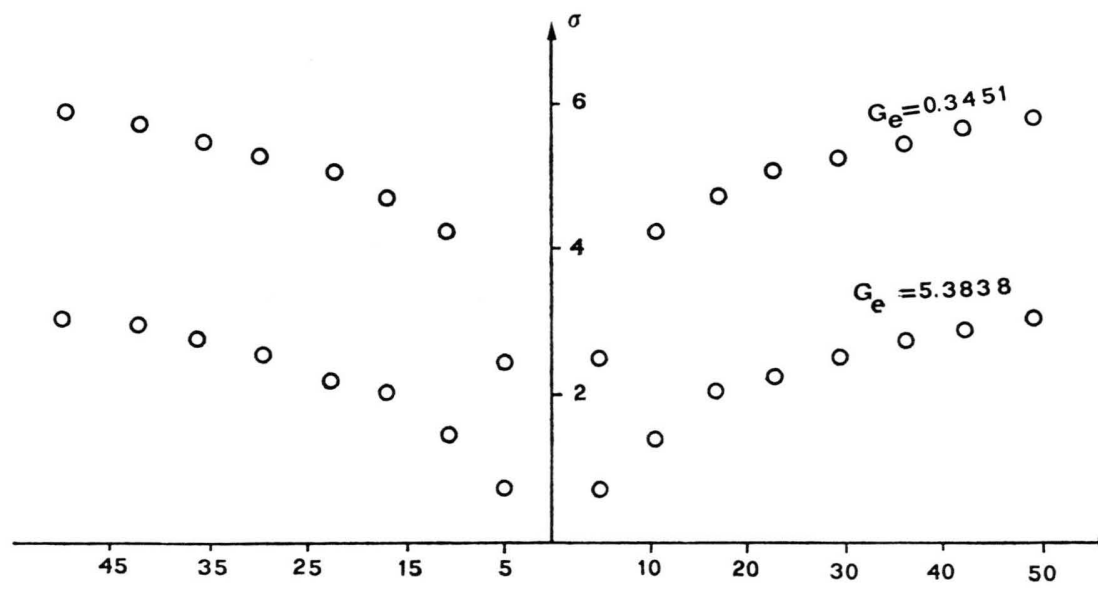

$\operatorname{Re}(\lambda) \leqq 0$. The location of the roots in the $n$th zonə, if $n$ becomes large, was predicted as

and

$$
\lim _{n \rightarrow \infty} \tau_{n}=\frac{\left(2 n+\frac{3}{2}\right) \pi}{\varepsilon_{k}}
$$

$$
\lim _{u \rightarrow \infty} \sigma_{n}=-\ln \frac{\left(2 n+\frac{3}{2}\right) \pi}{\xi_{k} h_{\text {in }} G\left(\varepsilon_{k}, \varepsilon_{0}\right)} .
$$

The forbidden regions and location of poles are shown in Figs. 1 and 2.

If the poles $i$ occur at $\lambda_{i}$, then the calculation of the residue of Eq. (11) leads to:

$$
\begin{aligned}
\psi(\varepsilon)= & G\left(\varepsilon_{k}, \varepsilon_{0}\right) \frac{I}{I+h_{\text {in }} G\left(\varepsilon_{k}, \varepsilon_{0}\right)} \\
& +\sum_{i=1}^{\infty} \frac{S\left(\lambda_{i}\right) e^{-\lambda_{i} \varepsilon}}{I+h_{\text {in }} G\left(\varepsilon_{k}, \varepsilon_{0}\right) \exp \left(-\lambda_{i} \varepsilon\right)} .
\end{aligned}
$$

The first term represents the asymptotic flux.

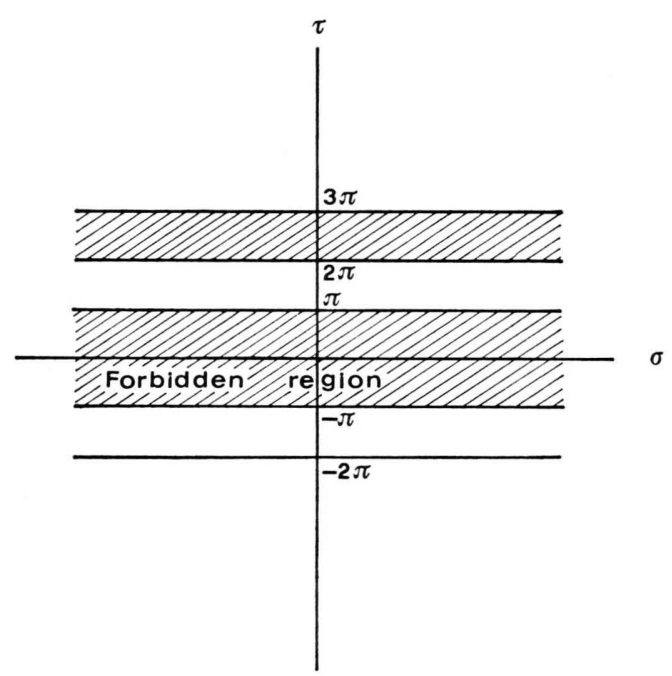

Fig. 1. Forbidden regions.

Fig $\cdot 2$. Location of the poles. 


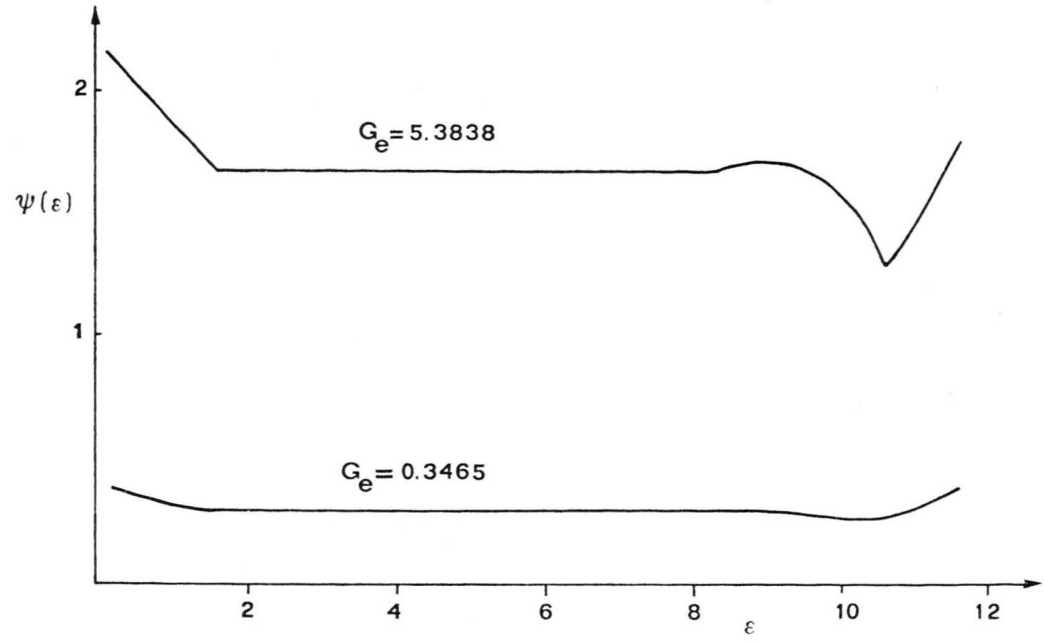

Fig. 3. Collision density for one inelastic level.

This last equation was used to calculate the collision density for the 922 and $865 \mathrm{keV}$ levels of $\mathrm{Fe}^{56}$ (Figure 3).

Application of the same procedure to the time dependent slowing down equation in an infinite homogeneous medium leads to

$$
\begin{aligned}
\psi(\varepsilon)=\frac{G\left(\varepsilon, \varepsilon_{0}\right)}{\sum_{\mathrm{t}}} \sum_{j} \frac{S\left(\lambda_{j}\right)}{I+h_{\mathrm{in}} G e^{-\lambda j}}[ & \frac{e^{-\alpha_{k} \varepsilon}}{-\lambda_{j}+h_{\mathrm{in}} G e^{-\lambda j}} \\
& \left.+\sum_{k} \frac{e^{-\alpha_{i} \varepsilon}}{\left(-\lambda_{j}+\alpha_{j}\right)\left(-I-h_{\mathrm{in}} G e^{-\alpha k}\right)+\left(-\frac{\alpha}{2}+h_{\text {in }} G e^{-\alpha_{k}}\right)}\right],
\end{aligned}
$$

where $\alpha_{k}$ is the pole of $\alpha-h_{\text {in }} G\left(\varepsilon_{k}, \varepsilon_{0}\right) e^{-\alpha \varepsilon}=0$.

The average time moment reduces in the asymptotic solution to the result obtained by Williams [11]:

$$
\langle t\rangle=\frac{1}{v \Sigma_{\mathrm{t}}} \frac{2}{h_{\mathrm{in}} G} .
$$

[1] W. M. Stacey, Jr., Nucl. Sci. Eng. 41, 381 (1970).

[2] D. Okrent, R. Avery, and H. Hummel, Proc. Intern. Conf. Peaceful uses At. Energy 5, 347 (1955).

[3] T. Mureley and I. Kaplan, Trans. Amer. Nucl. Soc. 8, 238 (1965).

[4] C. Syros, Nucleonic 8, 396 (1966), EUR 3457e (1967).

[5] F. Premuda, RT/IF 46 (1970).

[6] M. Segev, Nucl. Sci. Eng. 40, 424 (1970).

[7] T. D. Beynon, J. Nucl. Eng. 26, 177 (1972).
[8] S. A. El Wakil and E. A. Saad, Atke 24, 276 (1973).

[9] H. Volkin, J. Appl. Phys. 55, 83 (1954).

[10] F. Greuling and G. Goertzel, Nucl. Sci. Eng. 7, 69 (1960).

[11] M. M. R. Williams, The Slowing Down and Thermalization of Neutrons, North-Holland, Amsterdam 1966.

[12] N. Corngold and M. J. Lineberry, Nucl. Sci. Eng. 53, 153 (1974). 